\title{
Article \\ Wheat Type One Protein Phosphatase Participates in the Brassinosteroid Control of Root Growth via Activation of BES1
}

\author{
Mariem Bradai 1,2,3 ${ }^{(D}$, Vitor Amorim-Silva ${ }^{4}\left(\mathbb{D}\right.$, Nibras Belgaroui $^{2}$, Alicia Esteban del Valle ${ }^{4}$, \\ Marie-Edith Chabouté ${ }^{5}$, Anne-Catherine Schmit ${ }^{5}$, Rosa Lozano-Duran ${ }^{3}$ (D), Miguel Angel Botella ${ }^{4}(\mathbb{D}$, \\ Moez Hanin ${ }^{2}$ and Chantal Ebel ${ }^{2, * \text { (D) }}$
}

Citation: Bradai, M.;

Amorim-Silva, V.; Belgaroui, N.; Esteban del Valle, A.

Chabouté, M.-E.; Schmit, A.-C.;

Lozano-Duran, R.; Botella, M.A.;

Hanin, M.; Ebel, C. Wheat Type One

Protein Phosphatase Participates in the Brassinosteroid Control of Root Growth via Activation of BES1. Int. J. Mol. Sci. 2021, 22, 10424. https:// doi.org/10.3390/ijms221910424

Academic Editor: Jana Oklestkova

Received: 27 July 2021

Accepted: 10 August 2021

Published: 27 September 2021

Publisher's Note: MDPI stays neutral with regard to jurisdictional claims in published maps and institutional affiliations.

Copyright: (c) 2021 by the authors. Licensee MDPI, Basel, Switzerland. This article is an open access article distributed under the terms and conditions of the Creative Commons Attribution (CC BY) license (https:// creativecommons.org/licenses/by/ $4.0 /)$.
1 Laboratory of Biotechnology and Plant Improvement, Center of Biotechnology of Sfax, BP "1177", University of Sfax, Sfax 3018, Tunisia; mariem@psc.ac.cn

2 Plant Physiology and Functional Genomics Research Unit, Institute of Biotechnology of Sfax, BP “1175”, University of Sfax, Sfax 3038, Tunisia; nebrasbelgaroui@yahoo.fr (N.B.); moez.hanin@isbs.usf.tn (M.H.)

3 Shanghai Center for Plant Stress Biology, CAS Center for Excellence in Molecular Plant Science, Chinese Academy of Sciences, Shanghai 201602, China; lozano-duran@sibs.ac.cn

4 Departamento de Biología Molecular y Bioquímica, Instituto de Hortofruticultura Subtropical y Mediterranea "La Mayora", Universidad de Malaga-Consejo Superior de Investigaciones Científicas (IHSM-UMA-CSIC), Universidad de Málaga, Campus Teatinos, 29071 Málaga, Spain; vitorsergiosilva@gmail.com (V.A.-S.); aesteban@uma.es (A.E.d.V.); mabotella@uma.es (M.A.B.)

5 Institut de Biologie Moléculaire des Plantes, CNRS, Université de Strasbourg 12, rue du Général Zimmer, 67084 Strasbourg, France; marie-edith.chaboute@ibmp-cnrs.unistra.fr (M.-E.C.); anne-catherine.schmit@ibmp-cnrs.unistra.fr (A.-C.S.)

* Correspondence: chantal.ebel@isbs.usf.tn; Tel.:+216-74-871-816

\begin{abstract}
Brassinosteroids (BRs) play key roles in diverse plant growth processes through a complex signaling pathway. Components orchestrating the BR signaling pathway include receptors such as kinases, transcription factors, protein kinases and phosphatases. The proper functioning of the receptor kinase BRI1 and the transcription factors BES1/BZR1 depends on their dephosphorylation by type $2 \mathrm{~A}$ protein phosphatases (PP2A). In this work, we report that an additional phosphatase family, type one protein phosphatases (PP1), contributes to the regulation of the BR signaling pathway. Co-immunoprecipitation and BiFC experiments performed in Arabidopsis plants overexpressing durum wheat TdPP1 showed that TdPP1 interacts with dephosphorylated BES1, but not with the BRI1 receptor. Higher levels of dephosphorylated, active BES1 were observed in these transgenic lines upon BR treatment, indicating that TdPP1 modifies the BR signaling pathway by activating BES1. Moreover, ectopic expression of durum wheat TdPP1 lead to an enhanced growth of primary roots in comparison to wild-type plants in presence of BR. This phenotype corroborates with a down-regulation of the BR-regulated genes CPD and DWF4. These data suggest a role of PP1 in fine-tuning BR-driven responses, most likely via the control of the phosphorylation status of BES1.
\end{abstract}

Keywords: type one protein phosphatase; PP1; Brassinosteroids; signaling; root; meristem; BES1

\section{Introduction}

Brassinosteroids (BRs) are phytohormones involved in diverse developmental and physiological responses going from root development, cell division and cell elongation, to photomorphogenesis and seed germination (for review see [1,2]). These BR-triggered responses occur through a well-defined signaling pathway starting with BR perception by the extracellular LRR domain of the BRI1 (BRASSINOSTEROID-INSENSITIVE 1) receptor [3,4] at the cell surface together with its co-receptor BAK1 (BRI1-ASSOCIATED RECEPTOR KINASE 1/SERK3 (SOMATIC EMBRYOGENESIS RECEPTOR KINASE [1,5,6]. In BR signaling, several transphosphorylation events mediated through BSK1 (BRASSINOSTEROID-SIGNALLING 
KINASE1), CDG1 (CONSTITUTIVE DIFFERENTIAL GROWTH1) and BIN2 (BRASSINOSTEROID INSENSITIVE 2) kinases will lead to the activation of BSU1 (BRI1-SUPPRESSOR1) phosphatase, which acts to dephosphorylate and inactivate BIN2, the negative regulator of BR signaling. At low BR concentrations, BIN2 phosphorylates two homologous transcription factors, BZR1 (BRASSINAZOLE-RESISTANT1) and BR-INSENSITIVE-EMSSUPPRESSOR1 (BES1/BZR2) (hereafter referred to as BES1), and inhibits their nuclear localization and DNA-binding activity [7]. The cytoplasmic retention of the inactivated BES1/BZR1 is promoted by 14-3-3 protein binding (for review see [8]). At higher BR levels, along with the BIN2 inactivation, type 2A protein phosphatase PP2A activates BZR1/BES1 via their dephosphorylation to develop appropriate cell responses and attenuates cellular $\mathrm{BR}$ biosynthesis via a negative feedback mechanism down-regulating BR biosynthetic genes such as DWF4 and CPD [9,10]. In addition, PP2A can also reversibly inactivate BRI1 through dephosphorylation [11].

The Arabidopsis thaliana (hereafter referred to as Arabidopsis) genome encodes around 150 different protein phosphatases grouped in three major classes depending on their phospho-substrates (phospho-serine, phospho-tyrosine and double-specificity S/T phosphatases; for review see $[12,13])$. Type one protein phosphatases (TOPP/PP1) are particular serine/threonine protein phosphatases, since their phosphatase activity is driven by interactors that control their activity, substrate specificity or subcellular localization $[13,14]$. Arabidopsis contains nine TOPP isoforms (called TOPP1-9), while rice, Vicia faba and bread wheat have 5, 4 and 18 PP1s, respectively [13,15-17]. Beyond an extremely strong primary structure conservation, PP1s are characterized by their functional diversity as they participate in multiple biological processes such as cell cycle regulation, glucose metabolism, transcription, protein synthesis, plant development, light signaling and stress responses [12,13]. The analysis of the Arabidopsis dominant-negative mutant topp4-1 revealed a role of TOPP4 in the gibberellic acid (GA) pathway by DELLAs dephosphorylation, and its subsequent degradation by the $26 \mathrm{~S}$ proteasome [18] in stopping PIF5 (PHYTOCHROME-INTERACTING FACTOR5) degradation during light signaling [19], and in dephosphorylating PIN1 to regulate auxin gradient at the epidermis [20]. Lately, TOPP4 has also been shown to be involved in plant autoimmunity, probably by its binding to the NB-LRR receptor SUT1 [21,22]. TOPP1 reinforced by its interaction with Arabidopsis Inhibitor 2 (AtI2), considered as a negative regulator of TOPP activities [23], is involved in ABA signaling by inactivating SnRK2 kinase [24]. TOPP9 and TOPP8, also named, respectively, AUN1 and AUN2, have been shown to regulate cell wall integrity during pollen tip growth and root hair elongation [25]. In rice, the RSS1 (RICE SALT SENSITIVE 1) protein interacts with OsPP1a to control meristem maintenance upon salt stress $[17,26]$, and OsPP1a overexpression confers salinity tolerance to rice plants [27].

Functional characterization of PP1 in plants is hampered by high redundancy and versatility, and furthermore by the large diversity of PP1-interacting proteins (PIP) that are essential to drive substrate specificity, activity and/or subcellular localization [14,28]. The durum wheat TdPP1a (hereafter referred to as TdPP1) is part of a large multigenic family in wheat composed of 18 different genes located on the different subgenomes and is phylogenetically closely related to Arabidopsis TOPP4, with $88 \%$ identity at the protein level [16]. Being the only wheat PP1 characterized so far, TdPP1 was shown to be strongly expressed in durum wheat roots, an active phosphatase in vitro and to be able to interact with AtI2 [16], but still, its biological function in planta remains unknown.

Therefore, the current research aims to characterize the function of the durum wheat PP1, through its ectopic expression in Arabidopsis. Our results provide evidence for an original contribution of TAPP1 in root growth and BRs signaling via activation of the BES1 transcription factor.

\section{Results}

The durum wheat TdPP1 [16] was ectopically expressed in the Arabidopsis Col-0 ecotype under the control of the CaMV 35S promoter with a GFP C-terminal fusion. 
Three homozygous lines (TdPP1-GFP L7, TdPP1-GFP L10, TdPP1-GFP L13) with a single insertion based on the segregation of the selectable marker, were selected for assessing transgene expression by RT-qPCR and immunoblot analyses. TdPP1 is expressed in these three transgenic lines at various levels, with TdPP1-GFP L10 and TdPP1-GFP L13 being the highest and the lowest expressing lines, respectively (Figure 1a,b). Observations by confocal microscopy of TdPP1-GFP L10 roots showed that TdPP1 is localized ubiquitously within the cell (Figure 1c), as it was previously reported in Nicotiana bentamiana [16].

Phenotypical analyses showed that the three lines have significantly longer primary roots compared to wild-type (WT) seedlings (Figure 1d). Previous observations of Arabidopsis plants overexpressing TOPP4 reported greater aerial tissue size but no data on roots were described [18]. Optimal primary root growth and development is orchestrated by a complex, fine-tuned interplay of different phytohormones [29]. Among these, brassinosteroids (BR) promote root growth at different levels along the root [30]. In our case, when exposed to $100 \mathrm{nM}$ of 24-epibrassinolide (eBL), the three transgenic lines exhibited, indeed, $40 \%$ higher primary root length compared to WT (Figure 1d-f), concomitant to a $30 \%$ increase in fresh weight (Figure 1g).

It is well established that external application of BR can affect root growth in two contrasting ways, depending on the concentrations. While low BR concentrations $(\leq 0.04 \mathrm{nM} \mathrm{eBL})$ trigger root growth, concentrations higher than $0.04 \mathrm{nM}$ inhibit root growth [31]. This root growth arrest can be explained by the fact that high eBL concentrations might inhibit cell division in meristematic regions while promoting cell elongation and differentiation [31-33]. Therefore, we monitored meristem size in WT and the TdPP1 transgenic lines after an exposure to eBL ( 0.4 and $1 \mathrm{nM}$ ) because higher eBL concentrations $(100 \mathrm{nM})$ used previously (Figure 1) avoid precise observations of meristematic tissues due to intense root curling. We used also as a control the BR-insensitive bes1- $D$, a gain-of-function mutant where BES1 protein stability is increased leading to a constitutive activation of BR signaling [9], hence negatively affecting meristem size due to a premature cell cycle exit [31]. When grown on a control 1/2 MS medium, TdPP1 overexpressing lines (OE) had comparable meristem size to that of WT, while bes1-D had a shorter meristem size (Figure 2b,e). Treatment with 0.4 or $1 \mathrm{nM}$ of eBL did not further change the meristem size of the bes1- $D$ mutant. However, the meristem of WT seedlings was $20 \%$ shorter in the presence of $0.4 \mathrm{nM} \mathrm{eBL}$. Interestingly, in TdPP1 OE lines the meristem size was not affected by this eBL concentration (Figure 2c,e). Treatment with $1 \mathrm{nM}$ eBL, reduced even more significantly the meristem size of WT (up to $40 \%$ decrease vs the MS grown WT seedlings) while this reduction was limited to $20 \%$ in the meristems of the TdPP1 OE lines (Figure 2d,e). This result clearly indicates that the meristems of TdPP1 OE lines show a decreased sensitivity to BR. 

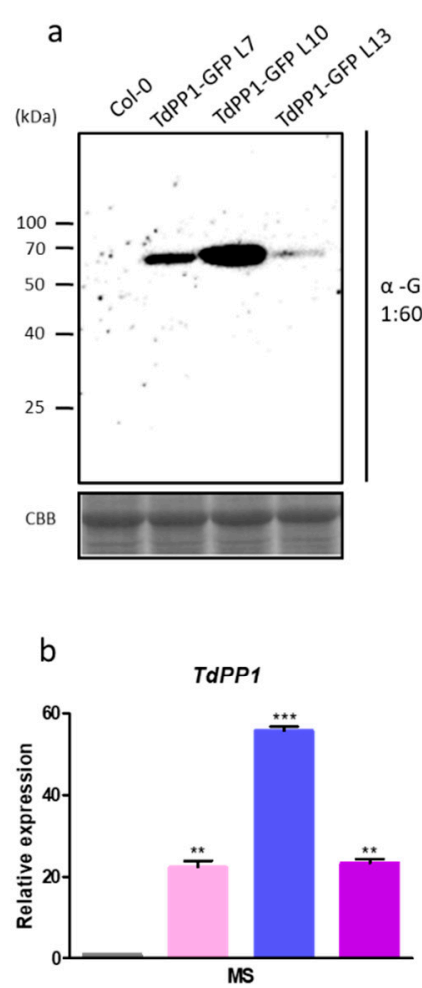

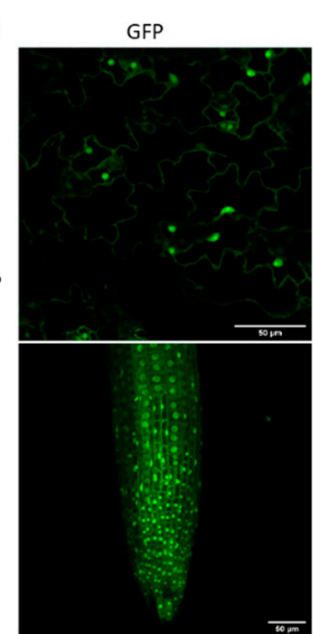

$\mathrm{f}$

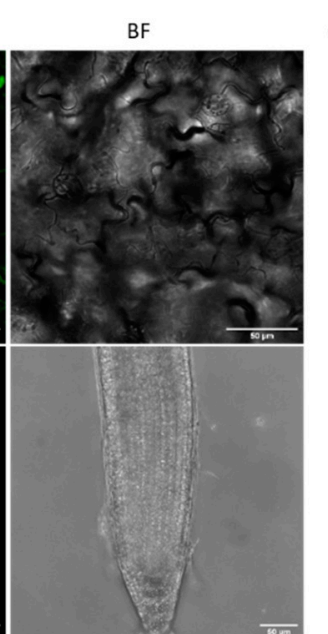
Col-0
TdPP1-GFP L7 TdPP1-GFP L10

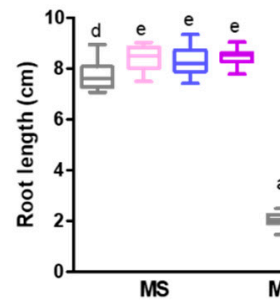

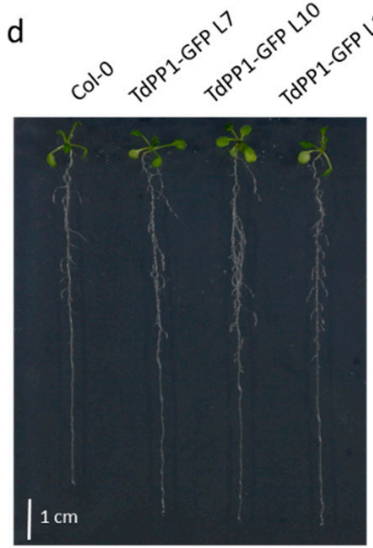

MS

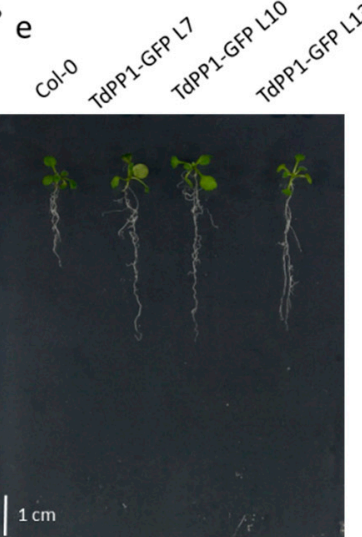

MS+ $100 \mathrm{nM}$ eBL

g

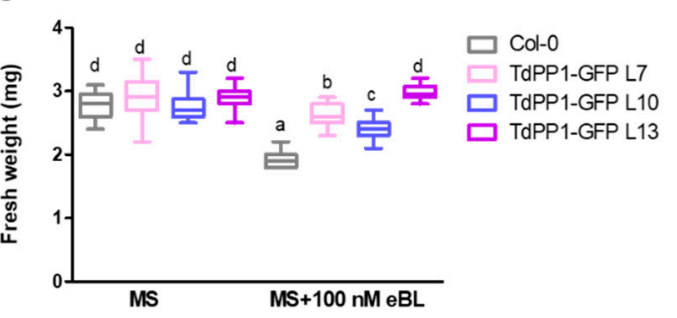

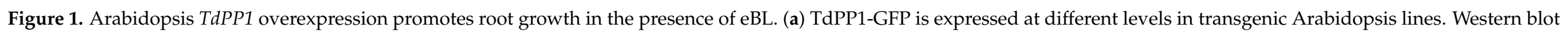

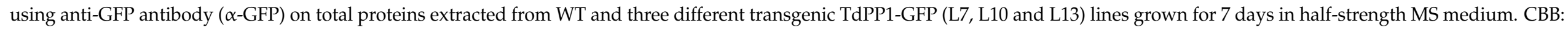

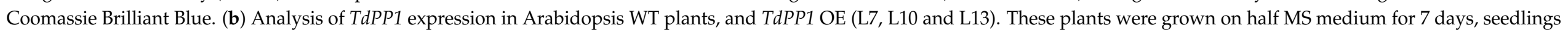

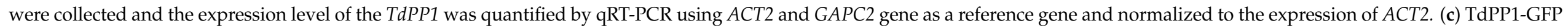

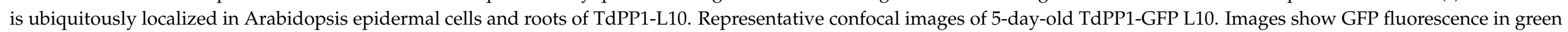

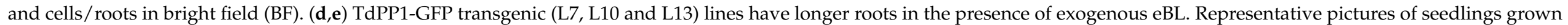

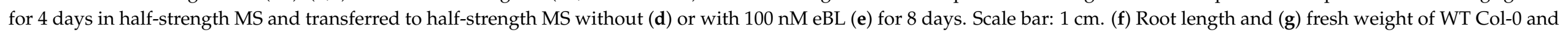

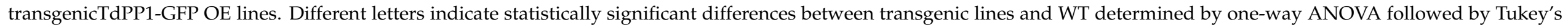

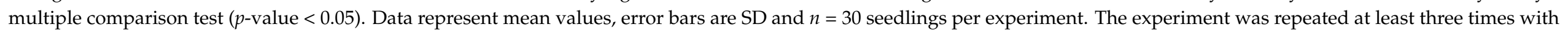
similar results. ${ }^{* *}: p<0.01,{ }^{* * *}: p<0.001$. 

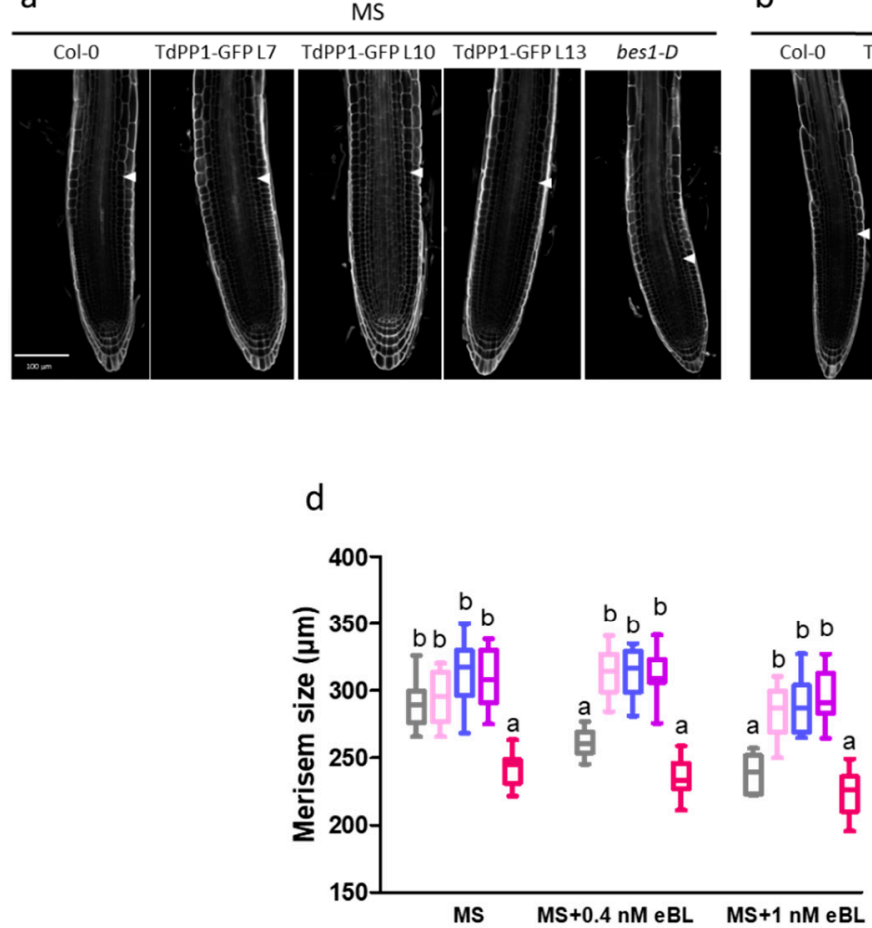

$\mathrm{S}+0.4 \mathrm{nM} \mathrm{eBL} \quad \mathrm{MS}+1 \mathrm{nM} \mathrm{eBL}$

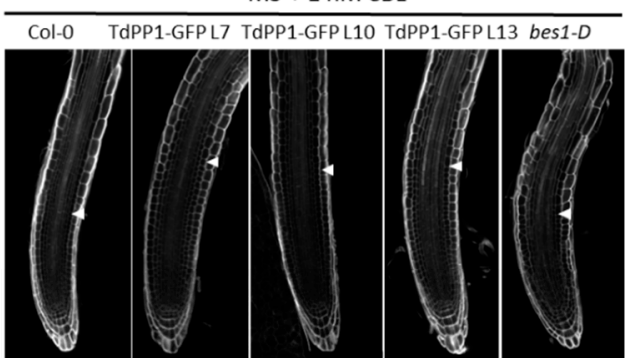

$\frac{\text { MS }+0.4 \mathrm{nM} \text { eBL }}{\text { Col-0 }}$ TdPP1-GFPL7 TdPP1-GFPL10 TdPP1-GFPL13 bes1-D
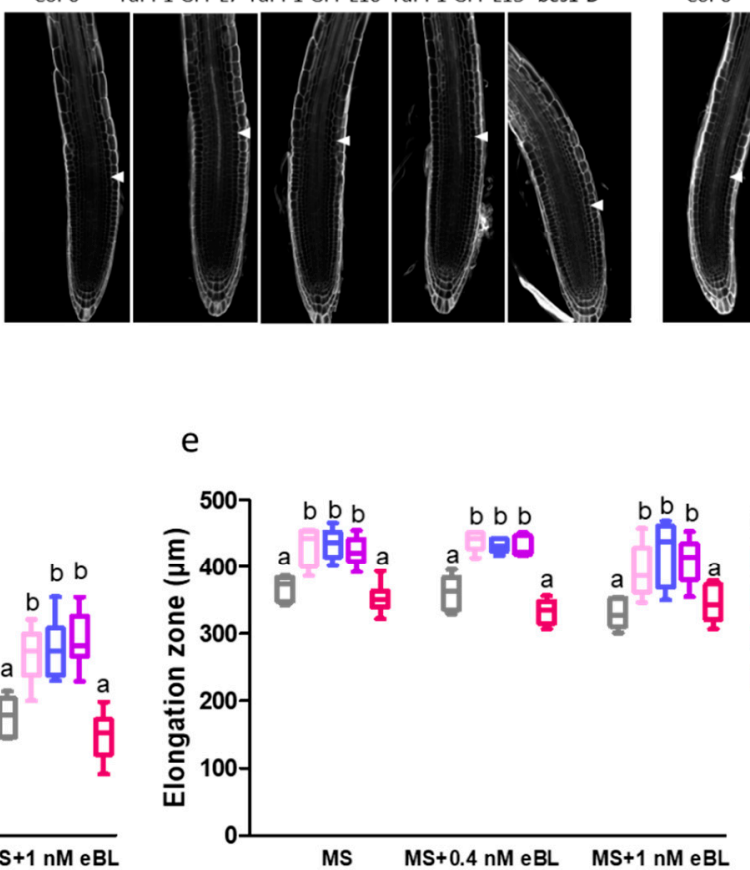

Col-0

TdPP1-GFP L7

$\square$ TdPP1-GFP L10

$\square$ TdPP1-GFP L13

$\square$ bes1-D

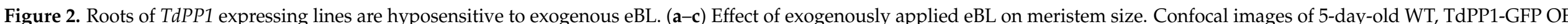

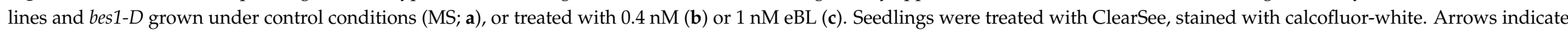

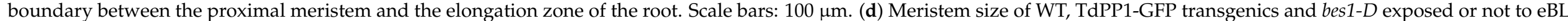

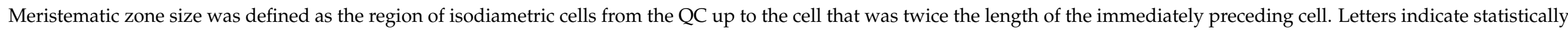

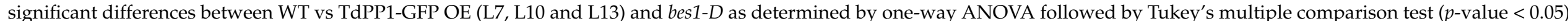

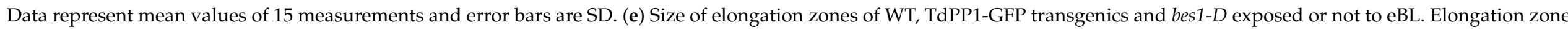

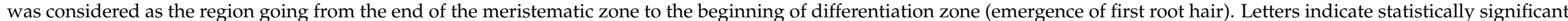

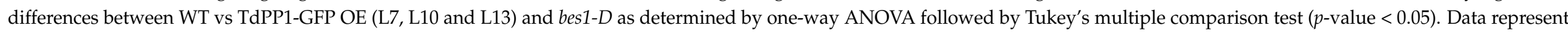
mean values of 15 measurements and error bars are $\mathrm{SD}$ 
We next compared the size of the elongation zones in the three TdPP1 OE lines with WT and bes1-D mutant (Supplementary Figure S1). Under control conditions, TdPP1 OE lines had elongation zones about $20 \%$ longer than that of WT and bes1-D. In the presence of $0.4 \mathrm{nM}$ and $1 \mathrm{nM}$ eBL, the elongation zone did not expand significantly in the TdPP1 overexpressors compared to WT (10\% increase), indicating again that these lines are less sensitive to exogenous BR.

To underpin the phenotype observed on the roots of the TdPP1 overexpressors, we investigated the expression of the BR-biosynthesis CPD and DWF4 genes that are negatively regulated at high $\mathrm{BR}$ concentrations. For this purpose, CPD and DWF4 expression analyses were performed by qRT-PCR in the presence and absence of BR, on the TdPP1-transgenic lines along with WT, and bes1-D as controls. Our results show that under control conditions, CPD expression was slightly, but significantly, down-regulated in TdPP1-OE lines, while DWF4 remained unchanged such as in WT (Figure 3). Treatment with eBL caused the downregulation of both CPD and DWF4 in WT seedlings (Figure 3), but this down-regulation was more pronounced in the TdPP1 OE lines. It, therefore, indicates that TdPP1 overexpression leads to an increased BR signaling.

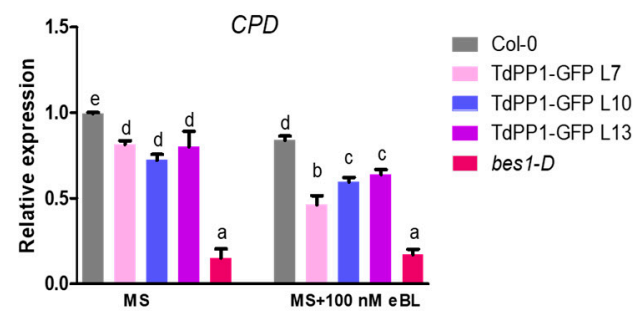

(a)

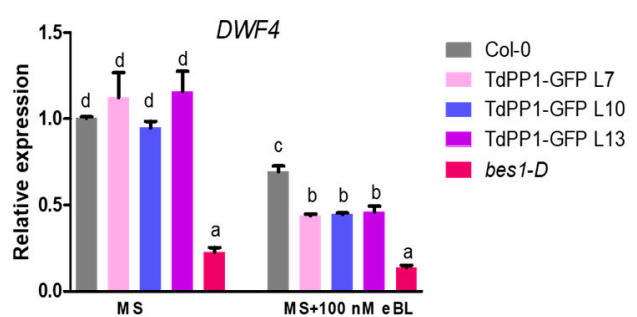

(b)

Figure 3. Relative expression of BR-biosynthesis genes CPD and DWF4. (a) Analysis of CPD and (b) DWF4, BR-responsive gene expression in Arabidopsis WT plants, TdPP1-GFP OE lines (L7, L10 and L13) and bes1-D. Plants were grown on half-strength MS medium for 7 days and transferred to half MS-strength liquid medium without or with $100 \mathrm{nM} \mathrm{BL}$ for $1 \mathrm{~h}$. Seedlings were collected and the expression level of CPD and DWF4 was quantified by qRT-PCR using the ACT2 and GAPC2 gene as reference genes. The expression of CPD and DWF4 was normalized to the expression of ACT2. Data represent mean values and error bars are SD from the average of three biological replicates (10 seedlings per biological replicate). Letters indicate statistically significant differences between WT vs TdPP1-GFP OE (L7, L10 and L13) and bes1-D as determined by one-way ANOVA followed by Tukey's multiple comparison test ( $p$-value $<0.05)$.

To get insight into the molecular mechanism by which TdPP1 contributes to BR signaling, we investigated whether TdPP1 is able to interfere with the activity of the BES1 transcription factor. BES1 is a key protein in BR signaling that is regulated by PP2A phosphatases [1] and its dephosphorylation allows its nuclear translocation and activation [7]. To this end, we examined the levels and phosphorylation status of BES1 in TdPP1 OE lines. Endogenous BES1 protein levels in WT and TdPP1 transgenic lines under control conditions and after treatment with $100 \mathrm{nM}$ eBL were analyzed by immunoblot using anti-BES1-specific antibody. As shown in Figure 4 (top panel), WT and TdPP1 OE seedlings showed similar levels of phosphorylated BES1 in control conditions. Interestingly, treatment with $100 \mathrm{nM}$ eBL increased the amount of dephosphorylated (active) BES1 in all three TAPP1 lines compared with WT. When we used an ultra-sensitive detection system on the same blot (Figure 4, bottom panel b), we could observe an increased accumulation of dephosphorylated BES1 in TdPP1 lines compared to WT seedlings already in control conditions. 


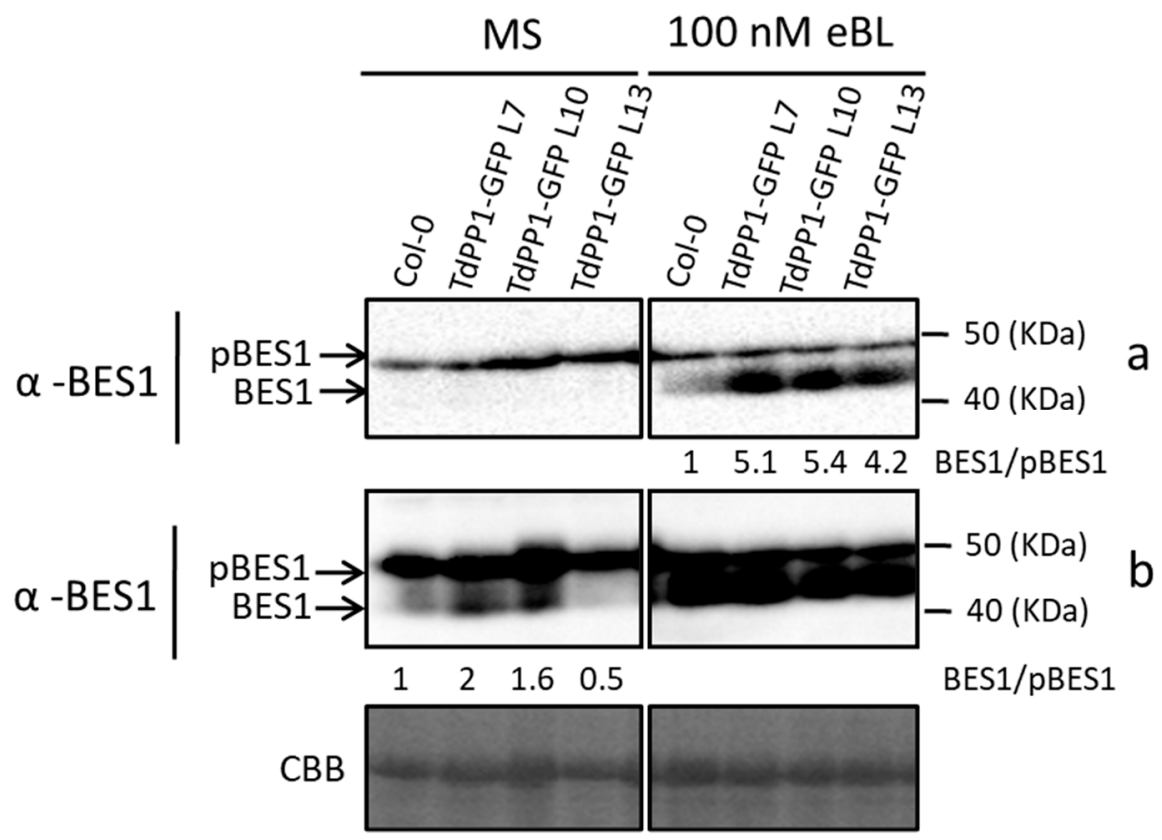

Figure 4. TAPP1 overexpression interferes with the balance pBES1/BES1. Phosphorylation status of BES1 in TdPP1-GFP OE lines in response to exogenously applied eBL. Protein extracts of 7-dayold seedlings (WT, L7, L10 and L13) treated or not for $1 \mathrm{~h}$ with $100 \mathrm{nM}$ eBL were analyzed by an immunoblot assay with a specific anti-BES1 antibody. Upper arrow indicates phosphorylated BES1 (pBES1) and lower arrow indicates dephosphorylated BES1 (BES1). (a) Standard exposure of the blot (b) exposure with increased sensitivity. CBB: Coomassie Brilliant Blue. Numbers below blots represent the ratio of unphosphorylated BES1 (BES1) to phosphorylated BES1 (pBES1).

Next, we investigated whether TdPP1 interacts with BES1, which could account for its higher dephosphorylation in the transgenic lines. For this purpose, we performed co-immunoprecipitation (Co-IP) assays with the TdPP1-GFP L10 line using anti-GFP-Trap beads (Figure 5a). As a control we used BRI1 because, as with BES1, it is also dephosphorylated by PP2A [11]. Our result shows that TdPP1-GFP co-immunoprecipitated endogenous BES1, but not BRI1. Surprisingly, TdPP1 only co-immunoprecipitated dephosphorylated BES1, despite its reduced proportion relative to the phosphorylated one in input (Figure 5a). These data strongly show that TdPP1 is able to bind to and might dephosphorylate BES1.

We then decided to verify this TdPP1-BES1 interaction in planta by bimolecular fluorescence complementation (BiFC assays) in N. benthamiana leaves. Co-expression of the TdPP1-C-terminal half of YFP (cYFP) with the BES1-N-terminal half of YFP reconstituted a functional YFP protein in the nucleus with strong signals observed in the nucleolus (Figure $5 b$ ). This is in agreement with the Co-IP data, since most of the dephosphorylated BES1 is localized in the nucleus [9]. Consistent also with the Co-IP experiment, no interaction was found between TdPP1 and BRI1 (Figure 5b) that interacts with BIK1 (BOTRYTIS-INDUCED KINASE 1), as previously shown [34]. Interestingly, in comparison to TdPP1's ubiquitous localization throughout the transgenic cells (Figure 1), the BiFC data also show that TdPP1 was able to relocate in the nucleus together with BES1. It is worth noting that TdPP1 interaction is restricted to unphosphorylated BES1, as observed by co-IP, suggesting that PP1-pBES1 interaction is highly labile or that dephosphorylation activity of PP1 is highly performant, leading to a rapid BES1-PP1-complex nuclear translocation, likely observed by BiFC. Therefore, our data strongly suggest that, as with PP2As, TdPP1 is able to bind BES1, thus causing its activation and nuclear translocation. 


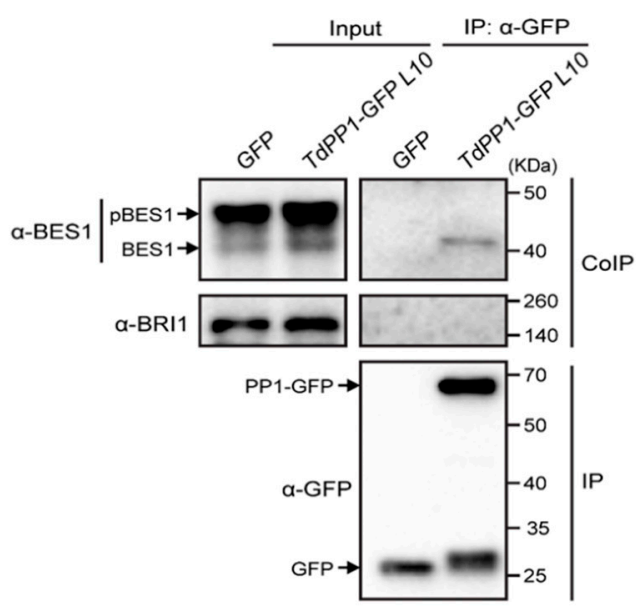

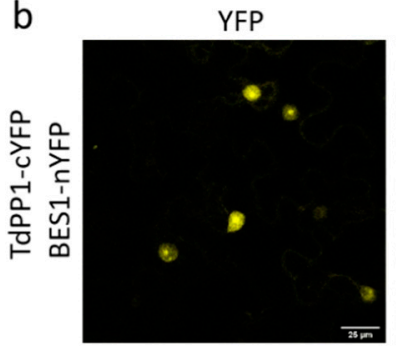

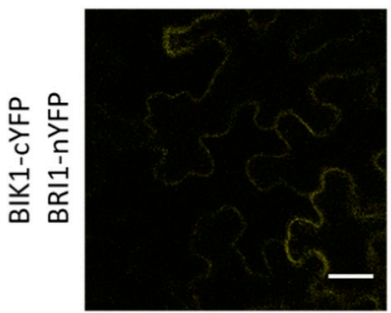

BF
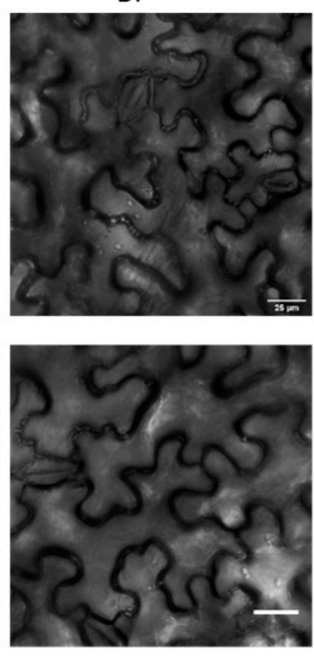
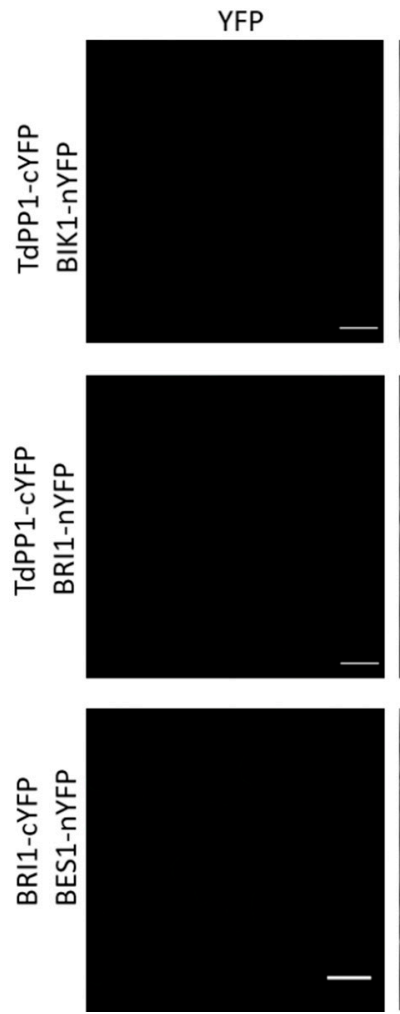

BF
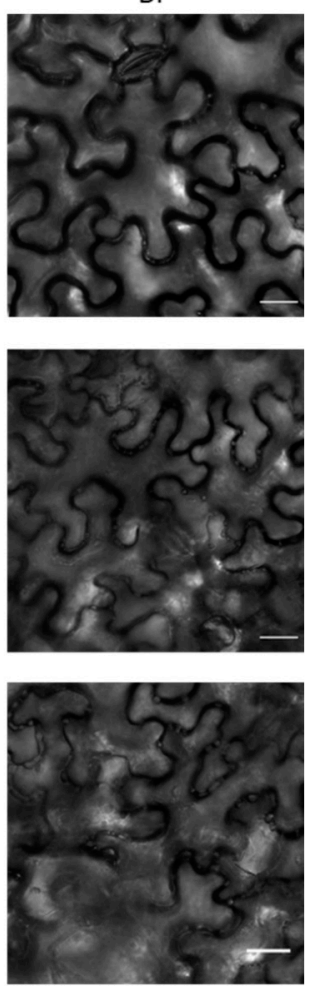

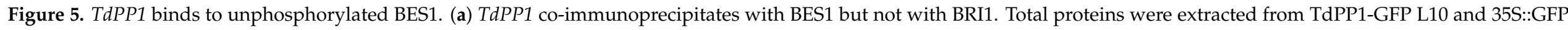

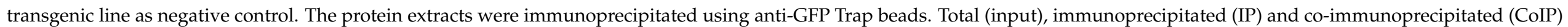

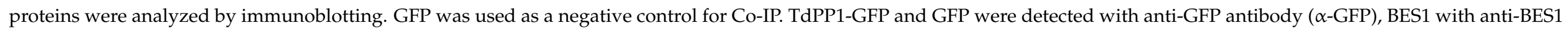

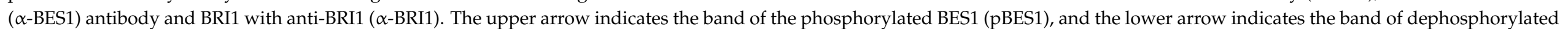

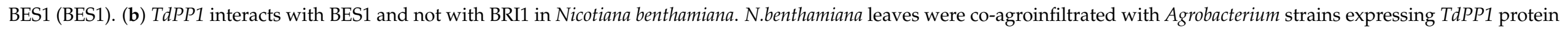

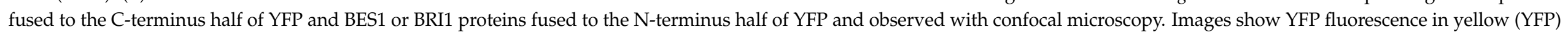

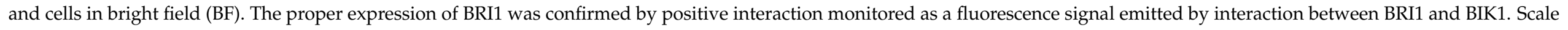
bars represent $25 \mu \mathrm{m}$. 


\section{Discussion}

Type one protein phosphatases are ubiquitous strongly conserved proteins with highly versatile activities still largely unknown in plants. Versatility is conveyed by high redundancy but also by their interaction with multiple regulators (PIPs) that modulate their activity or specify substrate binding or subcellular localization. The present study puts the light on a novel function for PP1s in promoting root growth through enhancing BR signaling. We showed here that wheat $T d P P 1$ contributes towards regulating $\mathrm{BR}$ signaling through activation of BES1.

Such a role in BR signaling pathway has never been reported so far for any plant PP1, in contrast to their known functions in other phytohormone signaling pathways. Indeed, Arabidopsis TOPP4 participates in GA signaling by dephosphorylation of DELLAs and in auxin transport by acting on PIN $[18,20]$. TOPP1 regulates ABA signaling by inhibiting SNF1-related protein kinases 2 (snRK2s; [24]), leading to partial release of the ABA-mediated root growth inhibition [24]. The latter example resembles to some extent our current observations, since besides PP2C, the main negative regulator of snRK2s [35,36], TOPP1 together with its regulatory partner AtI2, participates in snRK2s inhibition during ABA signaling [24]. TdPP1 may act besides the trimeric PP2A in regulating BES1 activity, hence adding complexity but also flexibility in the BR signaling pathway. However, our data showed that in contrast to PP2A, TdPP1 acts only on BES1 and has no visible effect on BRI1, suggesting that the wheat phosphatase may have a more subtle function in finetuning BR signaling. Alternatively, an association of TdPP1 to the PP2A complex replacing its catalytic subunit (as both show significant similarity) is not excluded. Depending on their expression features, subcellular localization and interaction with their regulatory partners, PP1s may contribute at different levels to modulate BR signaling. Extensive redundancy of PP1s and high modularity of PIP counter, unfortunately, the deciphering of their multiple roles in biological processes.

Adequate root growth and meristem maintenance result from a complex intervention of different phytohormones with contrasting outcomes on particular cell types or root zones. Phytohormones such as auxins, cytokinins and BR regulate each other's action, biosynthesis, transport and catabolism and have opposing effects on root growth [37,38]. The positive effect that TdPP1 exerted on root growth upon BR exposure may be due to its capacity to induce subtle changes in BR signaling in a way that helps the plant to cope with the negative effects of high BR concentrations on root growth and development. Moreover, BR biosynthesis and active BES1 nuclear accumulation optima overlap in the elongation zone, and little or no nuclear active forms of BES1 are found in the root tip [39-41]. By binding to and activating BES1, under control conditions, TdPP1 might slightly increase BR signaling through BES1 activation, all along the root, in a way that mimics optimal $\mathrm{BR}$ concentrations so that primary root growth is increased in these lines. Consistently also, the discrete down-regulation of CPD in the absence of BR may indicate that in untreated TAPP1 OE plants, BR signaling might already be increased. By adding exogenous high concentrations of $\mathrm{BR}, \mathrm{TdPP} 1$ overexpressing lines are partially insensitive because with already longer roots they were better prepared to withstand high BR concentrations. Despite the increased activation of BES1 in transgenic lines, these lines do not accurately phenocopy the dominant negative effect of bes1-D on BR response, showing that in these lines BES1 can return to an inactive state.

Importantly, the overexpression of TOPP4 leads to Arabidopsis plants with greater plant heights, enlarged hypocotyl and leaves, probably because of an increase in GA signaling and responses [18]. TdPP1-overexpressing plants did not exhibit greater above-ground tissues but had slightly longer roots compared to $\mathrm{WT}$, suggesting that TdPP1 overexpression may not perturb GA signaling. It is possible, though, that TdPP1 overexpression might affect the balance and signaling events of other hormonal pathways not yet observed, since BES1 is a hub for different hormonal signaling pathways, and that TdPP1 may act on other phytohormone signaling components. Although BR signaling mechanisms in Arabidopsis and rice are well documented, involvement of PP1s in regulating the BR pathway was 
not reported before. In wheat, the major BR signaling components are conserved but highly redundant, with each component having one copy per subgenome [42]. Therefore, uncovering this novel role of PP1s on BES1 activation would have been difficult in polyploid wheat.

Altogether, the data presented herein highlight a new role for plant PP1s in BR signaling and open up new perspectives in manipulating BR pathway for crop improvement.

\section{Materials and Methods}

\subsection{Plant Materials and Growth Conditions}

Wild type Arabidopsis Col-0 (seeds from the NASC Stock Center) together with bes1-D mutant [9] and transgenic lines generated in the present study were grown as follows. After seed surface sterilization and stratification for 3 days at $4{ }^{\circ} \mathrm{C}$, seedlings used for the brassinolide treatment, meristem size measurement, confocal imaging, quantitative RT-PCR (qRT-PCR) and phosphorylation status of BES1 were sowed on half strength Murashige et Skoog ( $1 / 2 \mathrm{MS}$ ) medium containing $1.5 \%$ sucrose and $0.8 \%$ agar. Plates were placed vertically or horizontally in a growth chamber with a photoperiod of $16 \mathrm{~h} \mathrm{light} / 8 \mathrm{~h}$ dark at $22{ }^{\circ} \mathrm{C}$ with $70 \%$ relative humidity. Nicotiana benthamiana seeds were sown directly on soil and cultured during 4 weeks at $22{ }^{\circ} \mathrm{C}$ with $70 \%$ relative humidity, with a photoperiod of $16 \mathrm{~h}$ light $/ 8 \mathrm{~h}$ dark.

\subsection{Transgenic Lines}

The TdPP1 ORF (KM203893) was PCR-amplified and cloned into the pENTR-D-TOPO vector using the pENTR Directional TOPO cloning kit (Invitrogen) as previously described by [16]. LR recombination was performed between the pENTR vector and the pB7FWG2 vector (Ref. [43], BASTA resistance) to create a C-terminal fusion of TdPP1 with eGFP. This construct was mobilized into the Agrobacterium tumefaciens GV3101 strain for Arabidopsis Col-0 transformation using the floral dip method [44]. Selection rounds until T3 generation were performed on BASTA $10 \mu \mathrm{g} / \mathrm{mL}$.

\section{3. eBL Treatment and Meristem Size Measurement}

For root length and fresh weight analyses, seedlings were grown vertically on $1 / 2$ MS medium for 4 days prior to transfer to a fresh medium containing either mock (ethanol $80 \%$ as eBL solvent) or $100 \mathrm{nM}$ eBL (PhytoTechnology Laboratories, Shawnee, KS, USA). After 8 days, seedlings were photographed and weighted. Root length was measured using Fiji [45] and graphs were performed using GraphPad Prism 6 (GraphPad Software, San Diego, CA, USA, www.graphpad.com). For meristem size, seedlings were grown for 5 days on $1 / 2$ MS medium containing either mock (ethanol $80 \%$ ), $0.4 \mathrm{nM}$ or $1 \mathrm{nM} \mathrm{eBL}$, then imaged and analyzed by confocal microscopy as described below.

\subsection{Phosphorylation Status of BES1}

Seedlings were grown vertically on $1 / 2$ MS medium for 7 days prior to transfer to liquid $1 / 2$ MS medium containing either ethanol $80 \%$ (eBL solvent) or $100 \mathrm{nM}$ eBL for $60 \mathrm{~min}$. Total proteins were extracted as described under "Extraction of Total Protein from Arabidopsis" and analyzed by immunoblotting using an anti-BES1 antibody (1:500; [46]) as described under "Western blotting".

\subsection{Western Blotting}

One hundred mg of one-week-old Arabidopsis TdPP1 OE seedlings were ground to powder in liquid nitrogen prior to the addition of $200 \mu \mathrm{L}$ of $2 \times$ Laemmli Buffer and boiled at $95^{\circ} \mathrm{C}$ for $10 \mathrm{~min}$. After $5 \mathrm{~min}$ centrifugation at top speed in a regular bench centrifuge, the supernatant was loaded on $10 \%$ SDS-PAGE polyacrylamide gel and proteins were separated by electrophoresis. Transfer to PVDF membrane (Immobilon-P, Millipore, Burlington, MA, USA) was performed during one hour at $18 \mathrm{~V}$ using the Trans Blot SD semi-dry transfer cell (BioRad, Hercules, CA, USA). After blocking in $1 \times$ TTBS, $5 \%$ skimmed milk for $2 \mathrm{~h}$ and 
three washes in $1 \times$ TTBS, the primary anti-GFP antibody was added at a dilution of 1:600 (mouse anti-GFP clone B-2; Santa Cruz Biotechnology, Dallas, TX, USA) and incubated overnight at $4{ }^{\circ} \mathrm{C}$. After three washes in $1 \times$ TTBS, the peroxidase-conjugated secondary antibody (anti-mouse IgG, 1/80,000; Sigma-Aldrich, St. Louis, MO, USA) was added during $1 \mathrm{~h}$ at room temperature. Detection was performed using the SuperSignal ${ }^{\circledR}$ West Femto Maximum Sensitivity Substrate (Thermo SCIENTIFIC, Waltham, MA, USA) and images were acquired at different exposure times with Chemi-doc XRS+ System (BioRad).

\subsection{Protein Extraction and Co-Immunoprecipitation (co-IP) Assays}

Co-immunoprecipitation was performed as previously reported [47]. Briefly, total proteins were extracted from $100 \mathrm{mg}$ of seedlings from Arabidopsis TdPP1 OE line 10 (TdPP1-GFP L10) in an extraction buffer $(2 \mathrm{~mL} / \mathrm{g}$ of powder; $50 \mathrm{mM}$ Tris- $\mathrm{HCl}, \mathrm{pH} 7.5$; $150 \mathrm{mM} \mathrm{NaCl} ; 10 \%$ glycerol; $10 \mathrm{mM}$ EDTA, pH 8; $1 \mathrm{mM} \mathrm{NaF} ; 1 \mathrm{mM} \mathrm{Na} \mathrm{MoO}_{4} \cdot 2 \mathrm{H}_{2} \mathrm{O}$; 10 mM DTT; 0.5 mM PMSF; $1 \%(v / v)$ P9599 protease inhibitor cocktail (Sigma); Nonidet P-40, CAS: 9036-19-5 (USB Amersham life science) $0,5 \%(v / v)$ ) at $4{ }^{\circ} \mathrm{C}$ for $30 \mathrm{~min}$ on constant end-over-end rocking. After a centrifugation $(9000 \mathrm{rpm} / \mathrm{min})$ for $20 \mathrm{~min}$ at $4{ }^{\circ} \mathrm{C}$, supernatants were filtered by gravity through Poly-Prep Chromatography Columns (\#7311550 Bio-Rad), and $100 \mu \mathrm{L}$ were saved as input for Western blot analysis. The remaining supernatants were incubated for $2 \mathrm{~h}$ at $4{ }^{\circ} \mathrm{C}$ with $15 \mu \mathrm{L}$ GFP-Trap coupled with agarose beads (Chromotek). During incubation of protein samples with GFP-Trap beads, the final concentration of detergent (Nonidet P-40) was adjusted to $0.2 \%(v / v)$ in all cases to avoid unspecific binding to the matrix, as recommended by the manufacturer. Following incubation, the beads were collected and washed four times with a wash buffer (extraction buffer but without detergent). Finally, beads were resuspended in $75 \mu \mathrm{L}$ of $2 \times$ Laemmli Sample Buffer and heated at $60{ }^{\circ} \mathrm{C}$ for $30 \mathrm{~min}$ to dissociate immunocomplexes from the beads. Total (input), immunoprecipitated (IP) and co-immunoprecipitated (CoIP) proteins were separated by electrophoresis in 10\% SDS-PAGE, and analyzed as described by Western blot analysis using anti-BRI1 (1:2000; [48]), anti-BES1 (1:500; [46]) or anti-GFP (1:600; Santa Cruz Biotechnology) antibodies. Appropriate peroxidase-conjugated secondary antibodies were added as follows: anti-mouse IgG whole molecule Peroxidase (1:80,000; catalog No. A9044, Sigma-Aldrich) for anti-GFP, and anti-rabbit IgG whole molecule Peroxidase (1:80,000; catalog No. A0545, Sigma-Aldrich) for anti-BRI1 and anti-BES1.

\subsection{Bimolecular Fluorescence Complementation (BiFC) Assays}

The DNA constructs used for agroinfiltration were in pDEST-GW-VYNE for a Nterminal fusion (BRI1-nYFP; BES1-nYFP) and pDEST-GW-VYCE (TdPP1-cYFP) for a Cterminal fusion with YFP [49]. N. benthamiana leaves were co-agroinfiltrated at an OD600 nm of 0.8 as previously described [47], with either TdPP1-cYFP and BRI1-nYFP, or TdPP1cYFP and BES1-nYFP. Leaves were observed by confocal microscopy (Leica TCS SP5 II) two days after co-infiltration.

\subsection{Real-Time Quantitative RT-PCR ( $q R T-P C R$ )}

For real-time quantitative RT-PCR (qRT-PCR), seedlings were grown as described above and total RNA was extracted using the Plant RNA kit (OMEGA Bio-tek, Norcross, GA, USA). cDNA was synthesized using the iScriptTM gDNA clear cDNA Synthesis Kit (Bio-Rad) according to the manufacturer's instructions. qPCR was performed in a BioRad CFX96 real-time system with HieffTM qPCR SYBR Green Master Mix (Yeason); $20 \mu \mathrm{L}$ of the PCR reaction mixture contained $10 \mu \mathrm{L}$ of SYBR Green mix (Bio-Rad, Hercules, CA, USA), $1 \mu \mathrm{L}$ of primer $\operatorname{mix}(10 \mu \mathrm{M}), 1 \mu \mathrm{L}$ reverse-transcribed product and $8 \mu \mathrm{L}$ of water. Details on gene-specific and normalizer (ACT2 and GAPC2, were used as normalizer) primers are indicated in Supplementary Table S1. Data were analyzed using the $2^{-\Delta \Delta \mathrm{CT}}$ method [50]. 


\subsection{Confocal Imaging of Arabidopsis and N. Benthamiana}

Confocal images were acquired using Leica TCS SP8 or Leica TCS SP5 II scanning confocal microscopes. To measure the meristem size, 5-day-old seedlings were fixed with $4 \%$ paraformaldehyde, then treated with ClearSee solution as previously described [51] and finally stained with calcofluor-white. The number of cortex cells in individual cell files was used to gauge the meristem size. The meristematic zone was defined as the region of isodiametric cells from the QC up to the cells that were twice longer than the immediately preceding cells. The settings used for the laser scanning are $405 \mathrm{~nm}$ excitation and detection at $425-475 \mathrm{~nm}$ for calcofluor-white and $514 \mathrm{~nm}$ excitation and detection at $525-570 \mathrm{~nm}$ for YFP. Images have been analyzed using Fiji [45].

\subsection{Statistical Analyses}

Statistical analyses were performed using GraphPad Prism6 software using $p$-values $<0.05$ and Tukey's multiple comparison test.

Supplementary Materials: The following are available online at https://www.mdpi.com/article/10 .3390/ijms221910424/s1, Figure S1: Elongation zones of TdPP1 transgenics are hyposensitive to high concentration of exogenous eBL, Table S1: List of primers used in the present study.

Author Contributions: M.B.: Investigation, Formal Analysis, Visualization, Writing—original draft preparation; M.-E.C. and A.-C.S.: Resources, Validation; V.A.-S.: Investigation, Formal Analysis, Visualization, Validation; A.E.d.V.: Investigation; N.B.: Investigation; R.L.-D.: Resources, Validation; M.A.B.: Methodology, Resources, Validation; M.H.: Resources, Writing-review and editing; C.E.: Project Administration, Methodology, Supervision, Validation, Writing—original draft preparation-review and editing. All authors have read and agreed to the published version of the manuscript.

Funding: The authors acknowledge the Tunisian Ministry of Higher Education and Research for funding, and the National Agency for Research Promotion (ANPR) for the financial management of the PRIMA Project GENDIBAR. This work was also supported by the Ministerio de Economía y Competitividad, co-financed by the European Regional Development Fund (grant BIO2017-82609-R to M.A.B and V.A-S). Mariem Bradai was granted by Mujeres por Africa and from COST INDEPTH Action (COST CA16212, 2017).

Institutional Review Board Statement: Not applicable.

Informed Consent Statement: Not applicable.

Data Availability Statement: No new data were created or analyzed in this study. Data sharing is not applicable to this article.

Acknowledgments: We thank Yanhai Yin and Michael Hothorn for generously providing the antiBES1 and the anti-BRI1 antibodies, respectively.

Conflicts of Interest: The authors declare no conflict of interest.

\section{References}

1. Belkhadir, Y.; Jaillais, Y. The molecular circuitry of brassinosteroid signaling. New Phytol. 2015, 206, 522-540. [CrossRef] [PubMed]

2. Sharma, I.; Kaur, N.; Pati, P.K. Brassinosteroids: A Promising Option in Deciphering Remedial Strategies for Abiotic Stress Tolerance in Rice. Front. Plant Sci. 2017, 8, 2151. [CrossRef]

3. Li, J.; Chory, J. A putative leucine-rich repeat receptor kinase involved in brassinosteroid signal transduction. Cell 1997, 90, 929-938. [CrossRef]

4. Wang, Z.Y.; Seto, H.; Fujioka, S.; Yoshida, S.; Chory, J. BRI1 is a critical component of a plasma-membrane receptor for plant steroids. Nature 2001, 410,380-383. [CrossRef]

5. Nam, K.H.; Li, J. BRI1/BAK1, a receptor kinase pair mediating brassinosteroid signaling. Cell 2002, 110, 203-212. [CrossRef]

6. Li, J.; Wen, J.; Lease, K.A.; Doke, J.T.; Tax, F.E.; Walker, J.C. BAK1, an Arabidopsis LRR receptor-like protein kinase, interacts with BRI1 and modulates brassinosteroid signaling. Cell 2002, 110, 213-222. [CrossRef]

7. Tang, W.; Yuan, M.; Wang, R.; Yang, Y.; Wang, C.; Oses-Prieto, J.A.; Kim, T.W.; Zhou, H.W.; Deng, Z.; Gampala, S.S.; et al. PP2A activates brassinosteroid-responsive gene expression and plant growth by dephosphorylating BZR1. Nat. Cell Biol. 2011, 13, 124-131. [CrossRef] 
8. Nolan, T.M.; Vukasinović, N.; Liu, D.; Russinova, E.; Yin, Y. Brassinosteroids: Multidimensional regulators of plant growth, development, and stress responses. Plant Cell 2020, 32, 295-318. [CrossRef]

9. Yin, Y.; Wang, Z.Y.; Mora-Garcia, S.; Li, J.; Yoshida, S.; Asami, T.; Chory, J. BES1 accumulates in the nucleus in response to brassinosteroids to regulate gene expression and promote stem elongation. Cell 2002, 109, 181-191. [CrossRef]

10. Wang, Z.Y.; Nakano, T.; Gendron, J.; He, J.; Chen, M.; Vafeados, D.; Yang, Y.; Fujioka, S.; Yoshida, S.; Asami, T.; et al. Nuclearlocalized BZR1 mediates brassinosteroid-induced growth and feedback suppression of brassinosteroid biosynthesis. Dev. Cell 2002, 2, 505-513. [CrossRef]

11. Wang, R.; Liu, M.; Yuan, M.; Oses-Prieto, J.A.; Cai, X.; Sun, Y.; Burlingame, A.L.; Wang, Z.-Y.Z.Y.; Tang, W. The BrassinosteroidActivated BRI1 Receptor Kinase Is Switched off by Dephosphorylation Mediated by Cytoplasm-Localized PP2A B' Subunits. Mol. Plant 2016, 9, 148-157. [CrossRef]

12. Farkas, I.; Dombrádi, V.; Miskei, M.; Szabados, L.; Koncz, C. Arabidopsis PPP family of serine/threonine phosphatases. Trends Plant Sci. 2007, 12, 169-176. [CrossRef]

13. Uhrig, R.G.; Labandera, A.M.; Moorhead, G.B. Arabidopsis PPP family of serine/threonine protein phosphatases: Many targets but few engines. Trends Plant Sci. 2013, 18, 505-513. [CrossRef]

14. Heroes, E.; Lesage, B.; Görnemann, J.; Beullens, M.; Van Meervelt, L.; Bollen, M. The PP1 binding code: A molecular-lego strategy that governs specificity. FEBS J. 2013, 280, 584-595. [CrossRef] [PubMed]

15. Takemiya, A.; Kinoshita, T.; Asanuma, M.; Shimazaki, K.-I. Protein phosphatase 1 positively regulates stomatal opening in response to blue light in Vicia faba. Proc. Natl. Acad. Sci. USA 2006, 103, 13549-13554. [CrossRef] [PubMed]

16. Bradai, M.; Mahjoubi, H.; Chini, A.; Chabouté, M.E.; Hanin, M.; Ebel, C. Genome wide identification of wheat and Brachypodium type one protein phosphatases and functional characterization of durum wheat TdPP1a. PLoS ONE 2018, 13, e0191272. [CrossRef]

17. Ogawa, D.; Morita, H.; Hattori, T.; Takeda, S. Molecular characterization of the rice protein RSS1 required for meristematic activity under stressful conditions. Plant Physiol. Biochem. 2012, 61, 54-60. [CrossRef] [PubMed]

18. Qin, Q.; Wang, W.; Guo, X.; Yue, J.; Huang, Y.; Xu, X.; Li, J.; Hou, S. Arabidopsis DELLA Protein Degradation Is Controlled by a Type-One Protein Phosphatase, TOPP4. PLoS Genet. 2014, 10, e1004464. [CrossRef]

19. Yue, J.; Qin, Q.; Meng, S.; Jing, H.; Gou, X.; Li, J.; Hou, S. TOPP4 regulates the stability of PHYTOCHROME INTERACTING FACTOR5 during photomorphogenesis in Arabidopsis. Plant Physiol. 2016, 170, 1381-1397. [CrossRef] [PubMed]

20. Guo, X.; Qin, Q.; Yan, J.; Niu, Y.; Huang, B.; Guan, L.; Li, Y.; Ren, D.; Li, J.; Hou, S. TYPE-ONE PROTEIN PHOSPHATASE4 Regulates Pavement Cell Interdigitation by Modulating PIN-FORMED1 Polarity and Trafficking in Arabidopsis. Plant Physiol. 2015, 167, 1058-1075. [CrossRef] [PubMed]

21. Liu, Y.; Yan, J.; Qin, Q.; Zhang, J.; Chen, Y.; Zhao, L.; He, K.; Hou, S. Type one protein phosphatases (TOPPs) contribute to the plant defense response in Arabidopsis. J. Integr. Plant Biol. 2019, jipb.12845. [CrossRef]

22. Yan, J.; Liu, Y.; Huang, X.; Li, L.; Hu, Z.; Zhang, J.; Qin, Q.; Yan, L.; He, K.; Wang, Y.; et al. An unreported NB-LRR protein SUT 1 is required for the autoimmune response mediated by type one protein phosphatase 4 mutation (topp4-1) in Arabidopsis. Plant J. 2019, 100, 357-373. [CrossRef]

23. Templeton, G.W.; Nimick, M.; Morrice, N.; Campbell, D.; Goudreault, M.; Gingras, A.-C.; Takemiya, A.; Shimazaki, K.; Moorhead, G.B.G. Identification and characterization of AtI-2, an Arabidopsis homologue of an ancient protein phosphatase 1 (PP1) regulatory subunit. Biochem. J. 2011, 435, 73-83. [CrossRef]

24. Hou, Y.J.; Zhu, Y.; Wang, P.; Zhao, Y.; Xie, S.; Batelli, G.; Wang, B.; Duan, C.G.; Wang, X.; Xing, L.; et al. Type One Protein Phosphatase 1 and Its Regulatory Protein Inhibitor 2 Negatively Regulate ABA Signaling. PLoS Genet. 2016, 12, e1005835. [CrossRef]

25. Franck, C.M.; Westermann, J.; Bürssner, S.; Lentz, R.; Lituiev, D.S.; Boisson-Dernier, A. The Protein Phosphatases ATUNIS1 and ATUNIS2 Regulate Cell Wall Integrity in Tip-Growing Cells. Plant Cell 2018, 30, 1906-1923. [CrossRef]

26. Ogawa, D.; Abe, K.; Miyao, A.; Kojima, M.; Sakakibara, H.; Mizutani, M.; Morita, H.; Toda, Y.; Hobo, T.; Sato, Y.; et al. RSS1 regulates the cell cycle and maintains meristematic activity under stress conditions in rice. Nat. Commun. 2011, 2, 278. [CrossRef] [PubMed]

27. Liao, Y.D.; Lin, K.H.; Chen, C.C.; Chiang, C.M. Oryza sativa protein phosphatase 1a (OsPP1a) involved in salt stress tolerance in transgenic rice. Mol. Breed. 2016, 36, 22. [CrossRef]

28. Bollen, M.; Peti, W.; Ragusa, M.J.; Beullens, M. The extended PP1 toolkit: Designed to create specificity. Trends Biochem. Sci. 2010, 35, 450-458. [CrossRef]

29. Jung, J.K.H.; McCouch, S. Getting to the roots of it: Genetic and hormonal control of root architecture. Front. Plant Sci. 2013, 4, 186. [CrossRef]

30. Ackerman-Lavert, M.; Savaldi-Goldstein, S. Growth models from a brassinosteroid perspective. Curr. Opin. Plant Biol. 2020, 53, 90-97. [CrossRef] [PubMed]

31. Gonzalez-Garcia, M.-P.; Vilarrasa-Blasi, J.; Zhiponova, M.; Divol, F.; Mora-Garcia, S.; Russinova, E.; Cano-Delgado, A.I. Brassinosteroids control meristem size by promoting cell cycle progression in Arabidopsis roots. Development 2011, 138, 849-859. [CrossRef]

32. Wei, Z.; Li, J. Brassinosteroids Regulate Root Growth, Development, and Symbiosis. Mol. Plant 2016, 9, 86-100. [CrossRef] [PubMed] 
33. Salazar-Henao, J.E.; Lehner, R.; Betegón-Putze, I.; Vilarrasa-Blasi, J.; Caño-Delgado, A.I. BES1 regulates the localization of the brassinosteroid receptor BRL3 within the provascular tissue of the Arabidopsis primary root. J. Exp. Bot. 2016, 67, 4951-4961. [CrossRef]

34. Lin, W.; Lu, D.; Gao, X.; Jiang, S.; Ma, X.; Wang, Z.; Mengiste, T.; Shan, L. Inverse modulation of plant immune and brassinosteroid signaling pathways by the receptor-like cytoplasmic kinase BIK1. Proc. Natl. Acad. Sci. USA 2013, 110. [CrossRef]

35. Leung, J.; Bouvier-Durand, M.; Morris, P.C.; Guerrier, D.; Chefdor, F.; Giraudat, J. Arabidopsis ABA response gene ABI1: Features of a calcium-modulated protein phosphatase. Science 1994, 264, 1448-1452. [CrossRef] [PubMed]

36. Meyer, K.; Leube, M.P.; Grill, E. A protein phosphatase 2C involved in ABA signal transduction in Arabidopsis thaliana. Science 1994, 264, 1452-1455. [CrossRef]

37. Pacifici, E.; Polverari, L.; Sabatini, S. Plant hormone cross-talk: The pivot of root growth. J. Exp. Bot. 2015, 66, 1113-1121. [CrossRef]

38. Wachsman, G.; Sparks, E.E.; Benfey, P.N. Genes and networks regulating root anatomy and architecture. New Phytol. 2015, 208, 26-38. [CrossRef]

39. Vukašinović, N.; Russinova, E. BRexit: Possible Brassinosteroid Export and Transport Routes. Trends Plant Sci. 2018, 23, 285-292. [CrossRef] [PubMed]

40. Vukašinović, N.; Wang, Y.; Vanhoutte, I.; Fendrych, M.; Guo, B.; Kvasnica, M.; Jiroutová, P.; Oklestkova, J.; Strnad, M.; Russinova, E. Local brassinosteroid biosynthesis enables optimal root growth. Nat. Plants 2021, 7, 619-632. [CrossRef]

41. Chaiwanon, J.; Wang, Z.Y. Spatiotemporal brassinosteroid signaling and antagonism with auxin pattern stem cell dynamics in Arabidopsis roots. Curr. Biol. 2015, 25, 1031-1042. [CrossRef] [PubMed]

42. Hou, L.; Zhang, A.; Wang, R.; Zhao, P.; Zhang, D.; Jiang, Y.; Diddugodage, C.J.; Wang, X.; Ni, Z.; Xu, S. Brassinosteroid Regulates Root Development with Highly Redundant Genes in Hexaploid Wheat. Plant Cell Physiol. 2019, 60, 1761-1777. [CrossRef] [PubMed]

43. Karimi, M.; Inzé, D.; Depicker, A. GATEWAY ${ }^{\mathrm{TM}}$ vectors for Agrobacterium-mediated plant transformation. Trends Plant Sci. 2002, 7, 193-195. [CrossRef]

44. Clough, S.J.; Bent, A.F. Floral dip: A simplified method for Agrobacterium-mediated transformation of Arabidopsis thaliana. Plant J. 1998, 16, 735-743. [CrossRef] [PubMed]

45. Schindelin, J.; Arganda-Carreras, I.; Frise, E.; Kaynig, V.; Longair, M.; Pietzsch, T.; Preibisch, S.; Rueden, C.; Saalfeld, S.; Schmid, B.; et al. Fiji: An open-source platform for biological-image analysis. Nat. Methods 2012, 9, 676-682. [CrossRef] [PubMed]

46. Yu, X.; Li, L.; Zola, J.; Aluru, M.; Ye, H.; Foudree, A.; Guo, H.; Anderson, S.; Aluru, S.; Liu, P.; et al. A brassinosteroid transcriptional network revealed by genome-wide identification of BESI target genes in Arabidopsis thaliana. Plant J. 2011, 65, 634-646. [CrossRef]

47. Amorim-Silva, V.; García-Moreno, Á.; Castillo, A.G.; Lakhssassi, N.; Del Valle, A.E.; Pérez-Sancho, J.; Li, Y.; Posé, D.; Pérez-Rodriguez, J.; Lin, J.; et al. TTL proteins scaffold brassinosteroid signaling components at the plasma membrane to optimize signal transduction in arabidopsis. Plant Cell 2019, 31, 1807-1828. [CrossRef]

48. Bojar, D.; Martinez, J.; Santiago, J.; Rybin, V.; Bayliss, R.; Hothorn, M. Crystal structures of the phosphorylated BRI1 kinase domain and implications for brassinosteroid signal initiation. Plant J. 2014, 78, 31-43. [CrossRef]

49. Gehl, C.; Waadt, R.; Kudla, J.; Mendel, R.R.; Hänsch, R. New GATEWAY vectors for high throughput analyses of protein-protein interactions by bimolecular fluorescence complementation. Mol. Plant 2009, 2, 1051-1058. [CrossRef]

50. Livak, K.J.; Schmittgen, T.D. Analysis of relative gene expression data using real-time quantitative PCR and the $2^{-\Delta \Delta C T}$ method. Methods 2001, 25, 402-408. [CrossRef]

51. Ursache, R.; Andersen, T.G.; Marhavý, P.; Geldner, N. A protocol for combining fluorescent proteins with histological stains for diverse cell wall components. Plant J. 2018, 93, 399-412. [CrossRef] [PubMed] 